\title{
A Robust $\boldsymbol{M}$-estimate Adaptive Equaliser For Impulse Noise Suppression
}

\author{
Zou Yuexian, Chan Shing-Chow and Ng Tung-Sung \\ Department of Electrical and Electronic Engineering \\ The University of Hong Kong, Pokfulam Road, Hong Kong \\ \{yxzou,scchan,tsng\}@eee.hku.hk
}

\begin{abstract}
In this paper, a FIR adaptive equaliser for impulse noise suppression is proposed. It is based on the minimization of an $M$-estimate objective function which has the ability to ignore or down-weight large error signal when it exceeds certain thresholds. An advantage of the proposed method is that its solution is governed by a system of linear equation, called the $M$-estimate normal equation. Therefore, traditional fast algorithms like the recursive least squares algorithm can be applied. Using a robust estimation of the thresholds and the recursive least square algorithm, an $M$-estimate RLS (M-RLS) algorithm is developed. Simulation results show that the proposed algorithm has better convergence performance than the N-RLS and MN-LMS algorithms when the input signal of the equaliser is corrupted by individually or consecutive impulse noises. It also shares the low steady state error of the traditional RLS algorithm.
\end{abstract}

\section{INTRODUCTION}

The adaptive equaliser is a kind of adaptive filter that has been developed to combat the channel distortions such as noise, spacing jamming and intersymbol interference over the last two decades [12]. Generally, for channel equalisation problem, noise is often considered as additive and Guassian. But a number of man-made or natural interference noises are impulsive in nature. Examples of impulsive process include switching transients in telephone channels, atmospheric noise in radio links and multiple access interference in radio communication networks [1,2]. Fig. 1 shows the block diagram of the channel equalization. The input to the equalizer is corrupted by additive Gaussian $\eta_{n}(n)$ as well as the impulse noise (outlier) $\eta_{i}(n) . s(n)$ is a random binary sequence transmitted through a linear dispersive channel, which is modelled as a time-invariant FIR filter with impulse response $c(i), i=1, \cdots, N_{c}, x_{c}(n)$ represents the channel output. The equaliser is implemented by an adaptive FIR filter with weight vector $\mathbf{w}(i)$. The convergence performance of conventional linear adaptive transversal filter will be significantly degraded by the impulse noise [10]. Recently, some results have been obtained for the large impulse noise suppression in system identification applications $[2,3,6,7]$. For instance, the mixed-norm adaptive filter using a combination of $L_{1}$ and $L_{2}$ norm as the objective function has been proposed recently $[2,3,4]$. Using the stochastic gradient method, a mixed-norm LMS (MN-LMS) algorithm, combining the Least Mean Square algorithm (LMS) and the Least Absolute Difference algorithm (LAD), is obtained [2]. Another class of nonlinear technique is to smooth out the momentary fluctuation of the error signal in conventional adaptive filters by means of some nonlinear clipping functions. These include the nonlinear LMS algorithm (N-LMS) and the nonlinear RLS algorithm (N-RLS) proposed in [6,7], respectively. In this paper, a $M$ estimate adaptive equaliser based upon the robust $M$ estimate cost function is proposed. $M$-estimate, like the median, belongs to the general class of robust statistical estimates, which are designed to perform robust estimation under model mismatch or the presence of outliners [9]. A block-based adaptive filter based on the Huber $M$-estimate objective function has previously been proposed in [11]. The Huber measure uses the $L_{2}$ norm when the signal error is smaller than a certain threshold and the $L_{1}$ norm when the error signal is large. The adaptive filter down-weights the outliners and behaves like a least squares filter on impulse free condition. The $M$-estimate proposed in this paper is differed from the Huber estimate in that the error will be completely ignored if the signal error is larger than certain threshold. A systematic method for estimating such thresholds is also proposed. The solution is governed by a system of linear equation similar to the conventional normal equation. Using this $M$-estimate normal equation, it is possible to derive different fast algorithms as in the traditional linear adaptive filters. In particular, a robust $M$-estimate recursive least square (M-RLS) adaptive algorithm is studied in this paper. Simulation results show that the proposed M-RLS algorithm is more robust against individual and consecutive impulse noise than the MN-LMS and the N-RLS algorithms. It also has a low steady state error similar to the RLS algorithm.

\section{OVERVIEW}

The most commonly used objective function in conventional linear adaptive equaliser is the least square error (LSE) which can be written as follows, 


$$
\min _{w(n)} J(n)=\sum_{k=1}^{n} \lambda^{n-k} e(k) e^{*}(k)
$$

where, $e(k)=d(k)-y(k)=d(k)-\mathbf{w}^{T}(n) \mathbf{X}(k) . d(k)$ and $y(k)$ are the desired output and actual output signal of the equaliser at time $k$. The forgetting factor $\lambda$ satisfies $0<\lambda \leq 1$. The minimisation of $J(n)$ in (1) results in the normal equation for solving the weight vector,

$$
\mathbf{R}(n) \hat{\mathbf{w}}(n)=\mathbf{r}(n),
$$

where, $\mathbf{R}(n)=\sum_{k=1}^{n} \lambda^{n-k} \mathbf{X}(k) \mathbf{X}^{T}(k)$ is the autocorrelation matrix and $\mathbf{r}(n)=\sum_{k=1}^{n} \lambda^{n-k} d(k) \mathbf{X}(k)$ is the cross correlation vector. A number of techniques have been proposed to solve (2) by recursively estimating the $\mathbf{R}^{-1}(n)$. Well-known techniques include the RLS and its various extensions [5]. When $x(n)$ is corrupted by impulse noise, $\mathbf{R}(n), \mathbf{r}(n), e(n)$ and hence $\hat{\mathbf{w}}(n)$ will exhibit momentary fluctuation which might take many iterations to recover. Such fluctuation will deteriorate the convergence performance of the adaptive equaliser. In what follows, a brief review of two recently proposed algorithms for combating impulse noise is given.

\section{N-RLS Algorithm [7]}

In this nonlinear recursive least square algorithm, a nonlinear clipping function, $f_{c}(\cdot)$, is applied to $e(n)$ to reduce its influence if $e(n)$ is large. More specifically, the weight update equation is

$$
\hat{\mathbf{w}}(n)=\hat{\mathbf{w}}(n-1)+f_{c}(e(n)) \mathbf{K}(n),
$$

where, $f_{c}(e(n))=\left\{\begin{array}{cc}e(n) & |e(n)| \leq h \\ h & |e(n)|>h\end{array}\right.$. The scalar parameter $h$ is estimated from the variance as follows,

$$
\begin{aligned}
& h=2.24 \hat{\sigma}_{e}(n), \\
& \quad \hat{\sigma}_{e}^{2}(n)=\lambda_{e} \hat{\sigma}_{e}^{2}(n-1)+\left(1-\lambda_{e}\right) e(n) e^{*}(n)
\end{aligned}
$$

where $0<\lambda_{e} \leq 1$ is a forgetting factor.

\section{Mixed-Norm LMS algorithm (MN-LMS) [2]}

In the mixed-norm adaptive filter, the weight vector is updated to minimize a combination of the $L_{1}$ and $L_{2}$ norms of the error signal. The resulting algorithm is similar to the combination of the well-known LMS and least absolute difference algorithm (LAD). The equation for updating the weight vector is given by,

$$
\begin{aligned}
\hat{\mathbf{w}}(n)=\hat{\mathbf{w}}(n-1) & +\mu\{\lambda(n) e(n) \\
& +(1-\lambda(n)) \operatorname{sign}(e(n)\} \mathbf{X}(n),
\end{aligned}
$$

where, $\mu$ is the step-size. $\lambda(n)$ is a mixing parameter which determines the relative contribution of the two norms to the objective function. It is estimated by

$$
\lambda(n)=2 \operatorname{erfc}\left(|e(n)| / \hat{\sigma}_{e}(n)\right),
$$

where, $\operatorname{erfc(.)}$ is the complementary error function. The variance $\hat{\sigma}_{e}(n)$ is estimated by the trimming window approach,

$$
\hat{\sigma}_{e}(n)=\sqrt{\mathbf{O}^{T}(n) \mathbf{A O}(n) /\left(N_{\sigma}-3\right)},
$$

where $\mathbf{A}=\operatorname{diag}(0,1,1, \cdots, 1,0)$ denotes a diagonal matrix and $\mathbf{O}(n)=\operatorname{sort}\left[e(n), \cdots, e\left(n-N_{\sigma}+1\right)\right]$. sort $[\cdots]$ is the operator for rearranging the components in square bracket in ascending order. In the presence of the impulse noise, $e(n)$ will be large and the weighting of the $L_{2}$ norm will be decreased to minimize the adverse effects of the impulse noise. The limitations of this algorithm are the slow convergence speed of its LMS-nature and the increased steady-error, due to the use of the mixed-norm.

\section{ROBUST $M$-ESTIMATE RLS ALGORITHM (M-RLS)}

In the proposed $M$-estimate RLS algorithm, instead of minimising the LSE, an $M$-estimate cost function is minimized,

$$
\begin{aligned}
& \min _{w(n)} J(n)=\sum_{k=1}^{n} \lambda^{n-k} \rho(e(k)), \\
& \rho(e(k))=\left\{\begin{array}{cc}
e(k)^{2} / 2 & |e(k)|<\xi \\
\xi|e(k)|-\xi^{2} / 2 & \xi \leq|e(k)| \leq \Delta \\
\Delta^{2} / 2 & |e(k)|>\Delta
\end{array}\right.
\end{aligned}
$$

where, $\rho(\cdot)$ is a modified $M$-estimator which is shown in Fig.5. For values of $|e(k)|$ smaller than $\xi$, the function is quadratic. For values of $|e(k)|$ in the interval $[\xi, \Delta]$, the function is linear. For values of $|e(k)|$ greater than $\Delta$, the function is equal to a constant $\alpha=\Delta^{2} / 2$. It becomes apparently that the $M$-estimate is capable of suppressing impulse noise with large amplitude. $\xi$ together with the parameter $\Delta$ controls the degree of suppression of the outliers. The smaller the ratio $\left(\alpha-\xi^{2} / 2\right) /(\Delta-\xi)$ is, the greater the suppression will be of the outliers. $\xi, \Delta$ and $\alpha$ are usually chosen according to the applications. $J(n)$, as defined as (6), helps to smooth out momentary fluctuation due to impulsive interferences. Substituting (7) in (6), we have,

$$
\begin{aligned}
\min _{w(n)} J(n)= & \sum_{\xi \leq|e(k)| \leq \Delta} \lambda^{n-k}\left\{\xi \operatorname{sgn}[e(k)] e(k)-\xi^{2} / 2\right\} \\
& +\frac{1}{2} \sum_{|e(k)|<\xi} \lambda^{n-k} e(k) e^{*}(k)+\sum_{|e(k)|>\Delta} \lambda^{n-k} \alpha .
\end{aligned}
$$


Setting $\dot{C}(n) / \dot{C} w(n)=0$ in (8) gives a system of linear equation in the $M$-estimate optimal weight vector $\tilde{\mathbf{w}}(n)$,

$$
\begin{aligned}
& \sum_{i=1}^{L} \tilde{w}_{i}(n) . \sum_{|e(k)|<\xi} \lambda^{n-k} x(n-i) x(n-k)= \\
& \xi \sum_{\xi \leq|e(k)| \leq \Delta} \lambda^{n-k} \operatorname{sgn}[e(k)] x(n-k)+\sum_{\mid e(k)]<\xi} \lambda^{n-k} d(n) x(n-k),(9)
\end{aligned}
$$

or in matrix form,

$$
\mathbf{R}_{N}(n) \tilde{\mathbf{w}}(n)=\mathbf{r}_{N}(n) .
$$

Equation (10), which is referred to be the $M$-estimate normal equation here, can be viewed as the counterpart of the normal equation (2). It should be noted that although the error function used is nonlinear, the solution of the weight vector is still linear. So, traditional adaptive filtering algorithms such as the RLS can also be used to solve (10) if $\mathbf{R}_{N}(n)$ and $\mathbf{r}_{N}(n)$ are appropriately updated.

Next, we consider the estimate of the scalar parameter $\xi$. For simplicity, we assume that the error signal except the outliers satisfies the Gaussian distribution with zero mean and variance $\hat{\sigma}_{e}^{2}$. Then, we can compute the probability of $e(n)$ greater than a given threshold $T$ as follows,

$$
\begin{gathered}
\theta_{T}(n)=\operatorname{Pr}\{|e(n)|>T\}=2 * \operatorname{Pr}\{e(n)>T\} \\
=2 *[1-\operatorname{Pr}\{e(n) \leq T\}]=1-\operatorname{erf}\left(\frac{T}{\sqrt{2} \hat{\sigma}_{e}}\right),
\end{gathered}
$$

where, $\operatorname{erf}(z)=\frac{2}{\sqrt{\pi}} \int_{0}^{z} \exp \left(-x^{2}\right) d x$ is the error function. $\operatorname{Pr}($.$) is the probability operator. The probability of e(n)$ greater than $\xi$ and $\Delta$ are therefore given by $\theta_{\xi}$ and $\theta_{\Delta}$, respectively. By appropriate choice of $\theta_{\xi}$ and $\theta_{\Delta}$, the values of $\xi$ and $\Delta$ can be determined based on the estimate value of $\hat{\sigma}_{e}$ from (11). In our work, $\theta_{\xi}$ and $\theta_{\Delta}$ are chosen as 0.2 and 0.1 , respectively so that we have $80 \%$ confident to down-weight the error in the interval $[\xi, \Delta]$ and $90 \%$ confident to reject it completely when $|e(n)|>\Delta$. Clearly, the choice of $\theta_{\xi}$ and $\theta_{\Delta}$ is a tradeoff between the convergence speed and the suppression of the outliers. Here, the variance, $\hat{\sigma}_{e}^{2}$, is estimated as [8],

$$
\begin{gathered}
\hat{\sigma}_{c}^{2}=T_{M A D}(n)=1.483 * \operatorname{med}\left(e^{2}(i)-\operatorname{med}\left(e^{2}(j)\right) \mid\right), \\
i, j=n, \cdots, n-N_{\sigma}+1 .
\end{gathered}
$$

where, $T_{M A D}(n)$ is the median absolute deviation from the median (MAD) of the block data in the data window, $N_{\sigma}$, and med $($.$) is the median operator. This approach$ can be viewed as the most $B$-robust estimator [8]. Based on above estimation for the parameters $\xi$ and $\Delta,(10)$ can be solved recursively. Depending on the values of $e(n)$, there are three different cases to consider.
Case 1: $|e(n)|<\xi$, in this case, the error function is quadratic which is similar to the conventional least square solution. Therefore $\mathbf{R}_{N}(n)$ and $\mathbf{r}_{N}(n)$ are updated as:

$$
\begin{aligned}
& \mathbf{R}_{N}(n)=\lambda \mathbf{R}_{N}(n-1)+\mathbf{X}(n) \mathbf{X}^{T}(n), \\
& \mathbf{r}_{N}(n)=\lambda \mathbf{r}_{N}(n-1)+d(n) \mathbf{X}(n) .
\end{aligned}
$$

It is natural to use fast adaptive algorithms, such as the Newton LMS, RLS or conjugate gradient algorithms, to update the filter weight recursively. Here, the conventional RLSA is adopted for its fast convergence speed.

Case 2: $\xi \leq|e(n)| \leq \Delta$, in this case, the $L_{1}$ norm of the error is used as the measure. From (9) and (10), it can be seen that $\mathbf{R}_{N}(n)$ should remains unchanged and the cross correlation vector should be updated as follows,

$$
\begin{aligned}
& \mathbf{R}_{N}(n)=\mathbf{R}_{N}(n-1), \\
& \mathbf{r}_{N}(n)=\mathbf{r}_{N}(n-1)+\xi \operatorname{sgn}(e(n)) \mathbf{X}(n) .
\end{aligned}
$$

Since $\mathbf{R}_{N}(n)$ is unchanged, the inverse in the previous iteration can be used to calculate the new weight vector,

$$
\tilde{\mathbf{w}}(n)=\mathbf{R}_{N}^{-1}(n-1) \mathbf{r}_{N}(n) .
$$

Case 3: $|e(n)|>\Delta$, in this case, the error will be completely ignored and updating is unnecessary.

$$
\begin{aligned}
& \mathbf{R}_{N}(n)=\mathbf{R}_{N}(n-1), \\
& \mathbf{r}_{N}(n)=\mathbf{r}_{N}(n-1), \\
& \tilde{\mathbf{w}}(n)=\tilde{\mathbf{w}}(n-1) .
\end{aligned}
$$

\section{SIMULATION RESULTS}

In this section, the performance of the various algorithms will be compared using two channel equalization experiments. In the experiments, the transmitted signal $s(n)$ is a random binary sequence with zero mean and variance one. The channel is assumed to be an FIR model with coefficients $[-0.0242,0.1786,0.8093,0.1786$, 0.0242 ]. The equaliser is modelled as a FIR system with $L=15$. The additive Gaussian noise $\eta_{n}(n)$ is assumed to be zero mean with variance $\sigma_{n}^{2}$. The signal to noise ratio at the equaliser input is defined by $S N R=20 \log 10\left(\sigma_{x_{c}}^{2} / \sigma_{n}^{2}\right)$, where $\sigma_{x_{c}}^{2}$ is the variance of the channel output signal. The impulse noise $\eta_{i}(n)$ is generated from the same multiplication model proposed in [2]. The initial weight vector of the equaliser is set to zero except the central one which set to one. The remaining parameters are set to $S N R=50 d b, \lambda=.99$, $\lambda_{e}=.9$, and $N_{\sigma}=L$ respectively. The mean square error (MSE) is used to evaluate the convergence performance and is attained by averaging over 100 independent runs. 
This simulation is set up to compare the convergence performance of the M-RLS, RLS, N-RLS and MN-LMS algorithms in individual impulse noise environment. The impulses are generated individually with arrival probability $\operatorname{Pa} \approx 2 * 10^{-3}$ and variance $A(n) \approx 200$, which occurs at $n=350,885$, respectively. The equaliser input signal $x(n)$ is plotted in Fig.2a. For N-RLS and MN-LMS, the weights are updated by (3) and (5a), respectively. $\mu$ in (5a) is chosen to be 0.025 . The parameters $h, \hat{\sigma}_{e}(n)$ and $\lambda(n)$ are estimated as described in section II for both algorithms. The MSE results are plotted in Fig.3. From Fig.3, it can be seen that for $n=1: 350$ the convergence rate of the M-RLS, N-RLS and RLS algorithms are identical with the same adaptation process when no impulse noise is present. When impulse noise is present in the input signal of equaliser at $n=350$ and $n=885$, the convergence of the algorithms are influenced greatly. From the curve (1) in Fig.3, we can see that M-RLS algorithm can overcome this effect in less than $L$ samples. But from curve (2), NRLS algorithm needs about 250 samples to eliminate the effect of the impulse noise. We also can see the impulse noise slows down the convergence speed of MN-LMS algorithm from curve (3). The curve (4) illustrates RLS algorithm is not robust and loses its good convergence ability in the impulse noise environment.

\section{Example 2}

The simulation is carried out to investigate the performance of the algorithms against successive impulse noise occurring in the input signal of the equaliser. The parameters are identical to those in experiment 1 except that the impulse noise arrives in successive from $n=350$ to 353 and an individual impulse occurs at $n=885$. The input signal of the equaliser $x(n)$ is plotted in Fig.2b. The MSE results are plotted in Fig.4. Curve (1) in Fig.4 clearly shows that successive impulse noise has very small influence on the convergence of the M-RLS algorithm. The convergence of the other three algorithms in Fig. 4 are significantly degraded as compared to that in Fig.3. The results illustrated the N-RLS, MN-LMS algorithms can not effectively suppress the successive impulse noise occurring at the input signal of the adaptive equaliser. The two simulation results demonstrate the better performance of the proposed M-RLS algorithm over the N-RLS and MN-LMS algorithms in suppressing individual and successive impulses occurring in the input signal of the adaptive equalizer.

\section{CONCLUSION}

In this paper, an $M$-estimate adaptive equaliser based on a robust modified $M$-estimate objective function is presented. The solution of the weight vector is governed by a system of linear equation similar to the conventional linear adaptive filters. Therefore, traditional techniques such the recursive least squares algorithm can be employed to solve the system recursively. A robust $M$ estimate recursive least squares algorithm (M-RLSA) is studied and methods for robust estimating the related parameters are derived. Simulation results demonstrate that the proposed M-RLS based equaliser has better convergence performance than the MN-LMS and the $\mathrm{N}$ RLS algorithm when individual and successive impulse noise is present in the equaliser input signal.

\section{REFERENCE}

(1) Blackard, K. L. etc. "Measurements and Models of Radio Frequency Impulsive Noise for Indoor Wireless Communications", IEEE J. Select. Areas Com., Vol. SAC-11, No. 7, pp 991-1001, Sept. 1993.

(2) Chambers, J. A. and Avlonitis, A. "A Robust MixedNorm (RMN) Adaptive Filter Algorithm", IEEE, Signal Processing Letters, Vol.4, No.2, pp 46-48. Feb., 1997.

(3) Chambers, J. A. etc. " Least Mean Mixed-Norm Adaptive Filtering ", Electronics Letters, Vol.30, pp 1574-1575, 1994.

(4) Tanrikulu, O. and Chambers, J. A. "Convergence and Steady-State Properties of the Least-Mean Mixed Norm (LMMN) Adaptive Algorithm", IEE Proc. Vision, Image and Signal Processing, Vol.143(3), pp 137-142, June 1996.

(5) Simon Haykin, Adaptive Filter Theory, Prentice Hall, $2^{\text {nd }}$ edition, 1991.

(6) Koile, S. "Adaptive Threshold Nonlinear Algorithm for Adaptive Filters with Robustness Against Impulsive Noise" Proceeding of ICASSP'96, Atlanta, pp 1644-1647, 1996.

(7) Leung, S. H and Weng, J. F. "Adaptive Nonlinear RLS Algorithm for Robust Filtering in Impulse Noise" IEEE Proceeding of ISCAS'97, pp 23372340, Hong Kong, June, 1997.

(8) Pitas, I. and Venetsanopoulos, A. N. Nonlinear Digital Filters Principles and Applications, Kluwer Academic Publisher, London.

(9) Hampel, Frank R., Ronchetti, E. M. and Stahel, W. A. Robust Statistics: the Approach Based on Influence Functions, John Wiley \& Sons, Inc. 1986.

(10)Qureshi, S. U. H. etc. "Adaptive Equalization" IEEE Proceedings Vol.73, No. 9, pp 1349-1387, 1985.

(11)Mili, L., Cheniae, M. G. and Rousseeuw, P. J. "Robust State Estimation Based on Projection Statistics", IEEE. Trans. On Power Systems, Vol. 11, No. 2, pp 1118-1126, May 1996.

(12) Prokias, John G. "Adaptive Equalization Techniques for Acoustic Telemetry Channels", IEEE Journal of Oceanic Eng., Vol. 16, No. 1, Jan. 1991. 


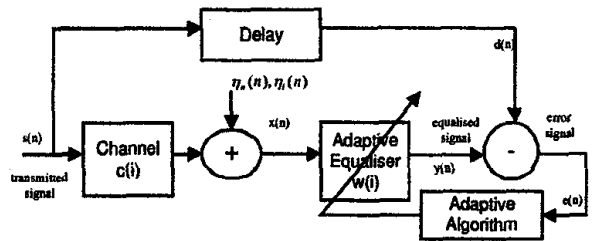

Fig.1 Adaplive Filtering Application-Channel Equalization Scheme
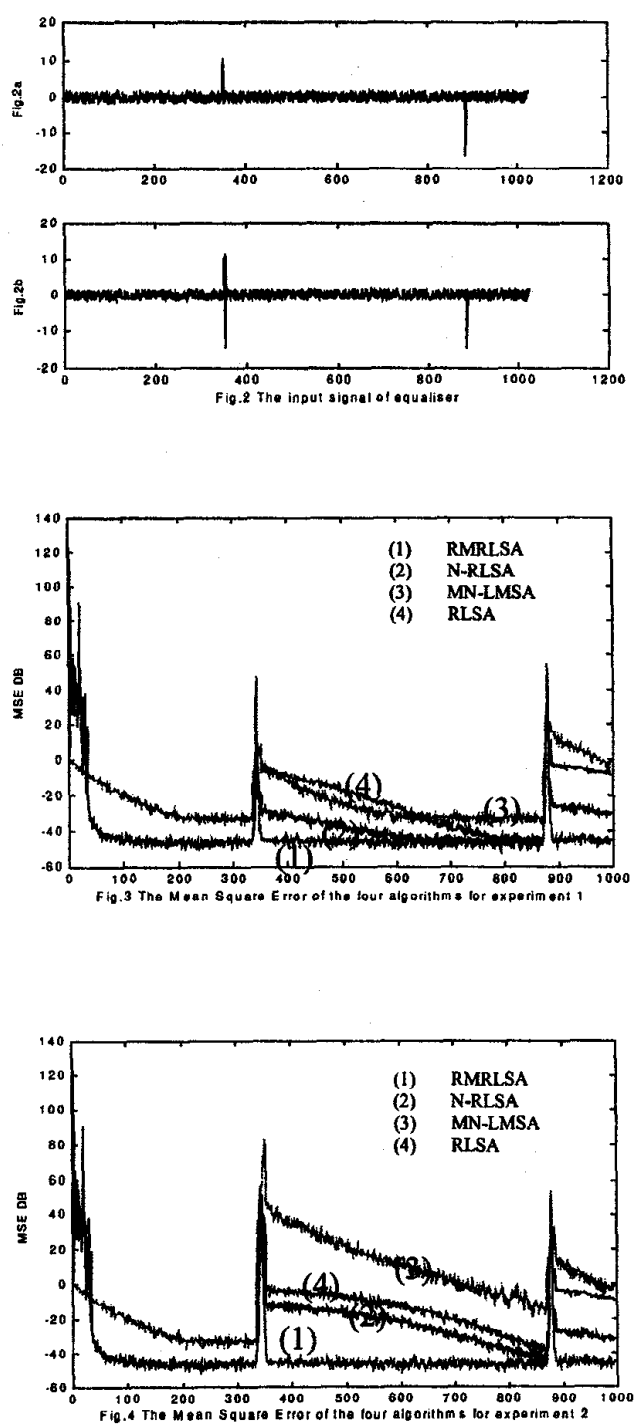

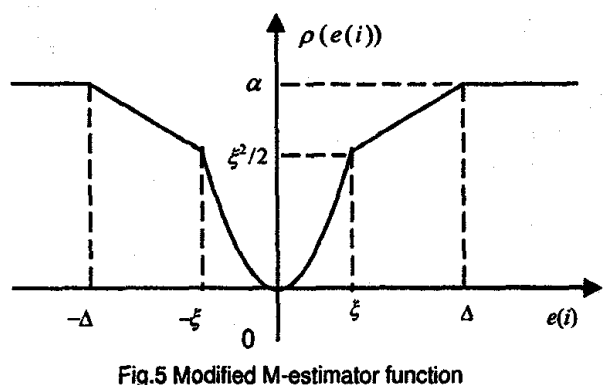

Fig.5 Modified M-estimator function 